\title{
Designing More Effective 9 Segment Display for Bengali and English Digits
}

\author{
Mohammad Badrul Alam Miah*, Md. Habibur Rahman, Md. Nazrul Islam
}

Department of Information \& Communication Technology, MBSTU, Tangail, BANGLADESH

*Corresponding Contact:

Email: badrul_ict05@yahoo.com

\begin{abstract}
Seven-segment display is well-known for displaying the English numerals form 0-9. In this paper 9-segment display for both Bengali and English digits have been proposed. Our proposed 9-segment display is more effective than the previously proposed 10-segment, 11-segment, 16-segment and 8-segment display for both Bengali and English as well as 9-segment, 10-segment and 18-segment display Bengali digits. It is an improvement of previously proposed segment display for both Bengali and English digits.
\end{abstract}

Key words

7-segment display, 9-segment display, Boolean function, Bengali and English digits

\section{INTRODUCTION}

Display with finite number of segment for each numeric character is preferred to dot matrix displays because the former saves both in memory space and cost. Seven segment display is commonly used for the display of English numerals. Both Bengali and English digits are represented using dot matrix, so the cost of display increases due to storage space, a large number of digits, power loss and design complexity. There are four proposed system for both Bengali and English numeric. Osiur et al (6th ICCIT, 2003b) use 11-segment, Khaled et al use 16 segment, Falzey et al (6th ICCIT, 2003a) use 8 segment and Sabbir Ahmed et al (Ahmed and Monira, 2004) use 10 segment for representing Bengali and English numeric. Here no segment intersects each other and it is easy to design and we can use only a single circuit for representing Bengali and English as well as two different circuits for this two-type numeric, here we show two different circuits for two different Bengali and English numeric.

\section{SEGMENTATION}

We have proposed 9-segment display for both Bengali and English numeric that is shown in Figure 1. 


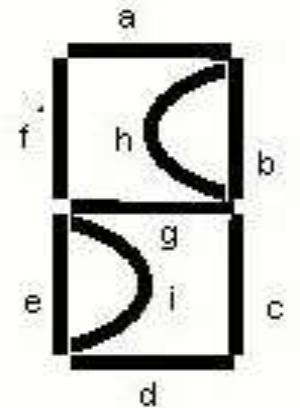

Figure 1: 9 segment displays

\section{Design OF Logic CiRcuits}

Both Bengali and English numeric system has 10 numeric from (0-9) so 4 bit inputs are used to represent each numeric. After analyzing which segments will be activated for which numeric, appropriate logic functions and circuits have derived in order to display each Bengali and English numeric. In following table I for Bengali and table II for English activated segment. Here 1 means activated and 0 means inactivated.

Table I: The activation state table of Bengali numeric

\begin{tabular}{|c|c|c|c|c|c|c|c|c|c|c|c|c|c|}
\hline Bengali numeric & $\mathrm{w}$ & $\mathrm{x}$ & $\mathrm{y}$ & $\mathrm{z}$ & $\mathrm{a}$ & $\mathrm{b}$ & $\mathrm{c}$ & $\mathrm{d}$ & $\mathrm{e}$ & $\mathrm{f}$ & $\mathrm{g}$ & $\mathrm{h}$ & $\mathrm{i}$ \\
\hline 0 & 0 & 0 & 0 & 0 & 1 & 1 & 1 & 1 & 1 & 1 & 0 & 0 & 0 \\
\hline 1 & 0 & 0 & 0 & 1 & 1 & 1 & 1 & 1 & 1 & 0 & 0 & 0 & 1 \\
\hline 2 & 0 & 0 & 1 & 0 & 1 & 1 & 0 & 1 & 1 & 0 & 1 & 0 & 0 \\
\hline 3 & 0 & 0 & 1 & 1 & 0 & 1 & 1 & 1 & 1 & 1 & 0 & 1 & 0 \\
\hline 4 & 0 & 1 & 0 & 0 & 1 & 1 & 1 & 1 & 1 & 1 & 1 & 0 & 0 \\
\hline 5 & 0 & 1 & 0 & 1 & 1 & 0 & 1 & 1 & 1 & 1 & 0 & 1 & 0 \\
\hline 6 & 0 & 1 & 1 & 0 & 0 & 0 & 1 & 1 & 1 & 1 & 0 & 1 & 0 \\
\hline 7 & 0 & 1 & 1 & 1 & 0 & 1 & 1 & 0 & 0 & 0 & 0 & 1 & 0 \\
\hline 8 & 1 & 0 & 0 & 0 & 0 & 0 & 0 & 0 & 1 & 1 & 1 & 0 & 1 \\
\hline 9 & 1 & 0 & 0 & 1 & 0 & 0 & 1 & 1 & 0 & 0 & 0 & 1 & 1 \\
\hline
\end{tabular}

Table II: The activation state table of English numeric

\begin{tabular}{|c|c|c|c|c|c|c|c|c|c|c|c|c|c|}
\hline English numeric & $\mathrm{w}$ & $\mathrm{x}$ & $\mathrm{y}$ & $\mathrm{z}$ & $\mathrm{a}$ & $\mathrm{b}$ & $\mathrm{c}$ & $\mathrm{d}$ & $\mathrm{e}$ & $\mathrm{f}$ & $\mathrm{g}$ & $\mathrm{h}$ & $\mathrm{i}$ \\
\hline 0 & 0 & 0 & 0 & 0 & 1 & 1 & 1 & 1 & 1 & 1 & 0 & 0 & 0 \\
\hline 1 & 0 & 0 & 0 & 1 & 0 & 1 & 1 & 0 & 0 & 0 & 0 & 0 & 0 \\
\hline 2 & 0 & 0 & 1 & 0 & 1 & 1 & 0 & 1 & 1 & 0 & 1 & 0 & 0 \\
\hline 3 & 0 & 0 & 1 & 1 & 1 & 1 & 1 & 1 & 0 & 0 & 1 & 0 & 0 \\
\hline 4 & 0 & 1 & 0 & 0 & 0 & 1 & 1 & 0 & 0 & 1 & 1 & 0 & 0 \\
\hline 5 & 0 & 1 & 0 & 1 & 1 & 0 & 1 & 1 & 0 & 1 & 1 & 0 & 0 \\
\hline 6 & 0 & 1 & 1 & 0 & 1 & 0 & 1 & 1 & 1 & 1 & 1 & 0 & 0 \\
\hline 7 & 0 & 1 & 1 & 1 & 1 & 1 & 1 & 0 & 0 & 0 & 0 & 0 & 0 \\
\hline 8 & 1 & 0 & 0 & 0 & 1 & 1 & 1 & 1 & 1 & 1 & 1 & 0 & 0 \\
\hline 9 & 1 & 0 & 0 & 1 & 1 & 1 & 1 & 1 & 1 & 0 & 1 & 0 & 0 \\
\hline
\end{tabular}




\section{A. Boolean Function for Bengali Numeric}

From the table-I we can write the following logic functions for different 9 segments in min term.

$$
\begin{aligned}
& a=\sum(0,1,2,4,5) \\
& b=\sum(0,1,2,3,4,7) \\
& c=\sum(0,1,3,4,5,6,7,9) \\
& d=\sum(0,1,2,3,4,5,6,9) \\
& \mathrm{e}=\sum(0,1,2,3,4,5,6,8) \\
& \mathrm{f}=\sum(0,3,4,5,6,8) \\
& \mathrm{g}=\sum(2,4,8), \\
& \mathrm{h}=\sum(3,5,6,7,9), \mathrm{i}=\sum(1,8,9)
\end{aligned}
$$

\begin{tabular}{|c|c|c|}
\hline Segments & Sum of Production & Simplified Logic function \\
\hline a & $\mathrm{a}=\sum(0,1,2,4,5)$ & $\bar{w} \bar{y}-w \times \bar{z}$ \\
\hline $\mathrm{b}$ & $\mathrm{b}=\sum(0,1,2,3,4,7)$ & $\bar{w} \bar{x}$ । wyz $-\bar{w} y z$ \\
\hline c & $\mathrm{c}=\sum(0,1,3,4,5,6,7,9)$ & $\bar{w} \bar{y}+\bar{w} z-\bar{w} x y+\bar{x} \bar{y} z$ \\
\hline $\mathrm{d}$ & $\mathrm{d}=\sum(0,1,2,3,4,5,6,9)$ & $\overline{\mathrm{w}} \overline{\mathrm{x}}+\mathrm{w} \overline{\mathrm{z}}+\mathrm{w} \overline{\mathrm{y}}+\overline{\mathrm{x}} \overline{\mathrm{y}} \mathrm{z}$ \\
\hline $\mathrm{e}$ & $\mathrm{e}=\sum(0,1,2,3,4,5,6,8)$ & $\bar{w} \bar{x}+w \bar{y} \mid w \bar{y}+\bar{x} \bar{y} z$ \\
\hline $\mathrm{f}$ & $\mathrm{f}=\sum(0,3,4,5,6,8)$ & $-\mathrm{wxz}+\overline{w x} \overline{\mathrm{y}}+\overline{\mathrm{x}} \overline{\mathrm{y}} \overline{\mathrm{z}}-\overline{\mathrm{w}} \overline{\mathrm{x}} \overline{\mathrm{y} z}$ \\
\hline $\mathrm{g}$ & $\mathrm{g}=\sum(2,4,8)$ & $\bar{w} x \bar{y} z-w \bar{x} \bar{y} \bar{z}+\bar{w} \bar{x} y \bar{z}$ \\
\hline $\mathrm{h}$ & $\mathrm{h}=\sum(3,5,6,7,9)$ & $\bar{w} y z \bar{w} x \bar{y}+\bar{w} x z \mid w x v z$ \\
\hline $\mathrm{i}$ & $\mathrm{i}=\sum(1,8,9)$ & $w \bar{x} \mid \bar{x} y$ \\
\hline
\end{tabular}

Table III: Simplified logic function for Bengali numeric

\section{B. Boolean Function For English Numeric}

From table-II we can write the following logic functions for different 9 segments in sum of production. For representing English numeric in our proposed 9 segment display 2 segments that are $h$ and i segments are totally unused.

$$
\begin{aligned}
& a=\sum(0,2,3,5,6,7,8,9) \\
& b=\sum(0,1,2,3,4,7,8,9) \\
& c=\sum(0,1,3,4,5,6,7,8,9) \\
& d=\sum(0,2,3,5,6,8,9) \\
& \mathrm{e}=\sum(0,2,6,8) \\
& \mathrm{f}=\sum(0,4,5,6,8,9) \\
& \mathrm{g}=\sum(2,3,4,5,6,8,9)
\end{aligned}
$$

Table IV: Simplified logic function for English numeric

\begin{tabular}{|c|l|c|}
\hline Segments & \multicolumn{1}{|c|}{ Sum of Production } & Simplified Logic function \\
\hline $\mathrm{a}$ & $\mathrm{a}=\sum(0,2,3,5,6,7,8,9)$ & $\overline{\mathrm{w}} \mathrm{y}+\overline{\mathrm{w}} \mathrm{xz}+\mathrm{w} \overline{\mathrm{x}}+\overline{\mathrm{x}} \mathrm{y} \overline{\mathrm{z}}$ \\
\hline $\mathrm{b}$ & $\mathrm{b}=\sum(0,1,2,3,4,7,8,9)$ & $\overline{\mathrm{w}} \overline{\mathrm{x}}+\overline{\mathrm{x}} \bar{y}+\overline{\mathrm{w}} \bar{y} \overline{\mathrm{z}}+\overline{\mathrm{w}} \mathrm{yz}$ \\
\hline $\mathrm{c}$ & $\mathrm{c}=\sum(0,1,3,4,5,6,7,8,9)$ & $\overline{\mathrm{x}} \bar{y}+\overline{\mathrm{w}} \mathrm{z}+\overline{\mathrm{w}} \mathrm{x}$ \\
\hline $\mathrm{d}$ & $\mathrm{d}=\sum(0,2,3,5,6,8,9)$ & $\overline{\mathrm{x}} \bar{y} \bar{z}+\overline{\mathrm{w}} \bar{x} y+\mathrm{w} \bar{x} \bar{y}+\overline{\mathrm{w}} \mathrm{y} \bar{z}$ \\
\hline $\mathrm{e}$ & $\mathrm{e}=\sum(0,2,6,8)$ & $\overline{\mathrm{w}} \mathrm{y} \bar{z}+\overline{\mathrm{x}} \bar{y} \bar{z}$ \\
\hline $\mathrm{f}$ & $\mathrm{f}=\sum(0,4,5,6,8,9)$ & $\overline{\mathrm{w}} \bar{y} \bar{z}+\overline{\mathrm{w}} \mathrm{x} \bar{y}+w \bar{x} \bar{y}+\bar{w} x \bar{z}$ \\
\hline $\mathrm{g}$ & $\mathrm{g}=\sum(2,3,4,5,6,8,9)$ & $\mathrm{w} \overline{\mathrm{x}} \bar{y}+\overline{\mathrm{w}} \mathrm{x} \bar{y}+\bar{w} \bar{x} y+\bar{w} y \bar{z}$ \\
\hline
\end{tabular}




\section{EXPERIMENT AND RESULT}

After performing the experiment we get the output that is shown in table-V

Table V: Representation of numeric of both Bengali and English by using our proposed 9segment display.

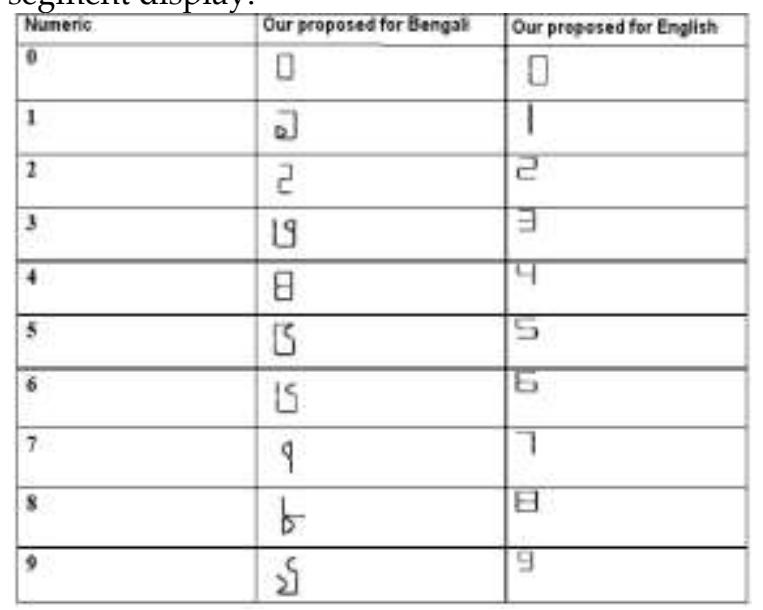

\section{CONCLUSION}

Drawbacks of previously proposed 8 segment's (6th ICCIT, 2003a) display quality and 10 (Ahmed and Monira, 2004), 11(6th ICCIT, 2003b) and 16 segment's number of segment, cost, complex design are overcome by our proposed system it's easy to implement any display device. Our proposed systems display quality is better and more effective than others. It is an improvement of 10 segment (Ahmed and Monira, 2004), 11-segment and 16 segment display. Though Fazley et al (6th ICCIT, 2003a) use 8 segment but its display quality is very poor and Sabbir Ahmed et al (Ahmed and Monira, 2004) use 10 segment but its display quality relatively poor (display quality is poor for Bengali 1 and 9 digits) than our proposed system. So our proposed 9 segment display is more effective and it has high quality display of Bengali and English digits.

\section{REFERENCES}

6th ICCIT 2003a, Jahangir Nagar University, page 338-341

6th ICCIT 2003b, JahangirNagar University, page 299-302

6th ICCIT 2003c Jahangir Nagar University, page 119-125

6th ICCIT 2003d Jahangir Nagar University, page 161-164

Ahmed Yousuf Saber, Mamun Al Murshed, Chowdhury Suman Ahmed, Chowdhury Mofizur Rahman, Designing 11-segment display for Bengali digits, P096 ICCIT 2002.

Barry M. Cook, Neil H. White, Computer Peripherals, pp.43-44, Edward Arnold (Publisher), 3rd Edition, 2002

ICECE 2004, DESIGNING A 24-SEGMENT DISPLAY FOR BENGALI NUMERICAL DIGITS AND CHARACTERS, PAGE 549-552

M. Morris Mano, Digital logic and computer design, pp.137-139, 189-191, 248-252, Prentice Hall,1995 Md. Rafiquzzaman, Microprocessors and Microcomputer based system design, 2nd Edition

Sabbir Ahmed, Serazam Monira, Designing a 10 segment display for Bangla and English numerals, 7th ICCIT 2004 BRACK University,page602-605

$$
--0--
$$

Online Archive Link: https://abc.us.org/ojs/index.php/ei/issue/archive 